\title{
EVALUACIÓN DE CUATRO ESPECIES FORESTALES ASOCIADAS CON CAFÉ (Coffea Arabica L.) Y EN MONOCULTIVO EN EL LITORAL ECUATORIANO
}

\author{
Pedro Suatunce Cunuhay ${ }^{1,2}$, Gorki Díaz Coronel ${ }^{1,3} \mathrm{y}^{\bullet}$ Luz García Cruzatty $^{1}$ \\ ${ }^{1}$ Unidad de Investigación Cientifica y Tecnológica, Universidad Técnica Estatal de Quevedo, $\mathrm{km} 1$ 11/2 vía \\ Quevedo-Santo Domingo de los Tsáchilas, C.P. 73. Quevedo, Los Ríos, Ecuador. ${ }^{1}$ luzcecilia29@hotmail.com \\ ${ }^{2}$ Facultad de Ciencias Ambientales, Universidad Técnica Estatal de Quevedo, $\mathrm{km} 1$ 11/2 vía a Santo Domingo \\ de los Tsáchilas, C. P. 73. Quevedo, Los Ríos, Ecuador. \\ ${ }^{3}$ Facultad de Ciencias Pecuarias, Universidad Técnica Estatal de Quevedo, km 7 vía a El Empalme \\ Mocache, Los Ríos, Ecuador
}

\begin{abstract}
RESUMEN
Q e e implantó un ensayo de sistema agroforestal con café (Coffea arabica L.) en el área del cantón Quevedo, en la Finca Experimental "La Represa", en el año de 1997. Se plantaron cuatro especies forestales en asociación con café ( $C$. arabica L.), y también se establecieron parcelas de árboles y café en monocultivo, en parcelas contiguas. Las especies forestales utilizadas fueron el guayacán blanco (Cybistax donnell-smithii Rose), laurel prieto (Cordia megalantha Chadat), fernansánchez (Triplaris cumingiana Wedd) y teca (Tectona grandis L. F.). El objetivo fue comparar la producción de madera y café bajo los sistemas agroforestales y en los sistemas de plantación en monocultivo tanto de los árboles como del café. La producción por hectárea de café fue mayor en monocultivo. La incidencia (\%) del minador del café (Perileucoptera coffeella Green) fue baja en los dos sistemas, además no hubo diferencias significativas entre los cafetales asociados y en monocultivo. El volumen de las especies forestales fue mayor en los sistemas agroforestales. Según la evaluación del uso eficiente de la tierra, cualquiera de los sistemas agroforestales evaluados es mejor que los cultivos puros de estas especies.
\end{abstract}

Palabras claves: Asociación, monocultivo, especies forestales, café.

\begin{abstract}
A $\mathrm{n}$ agroforestry system trial with coffee (Coffea arabica L.) was implanted in "La Represa" Farm, nearby Quevedo on 1997. Four forest species associated with coffee and it was also established plots of coffee and trees in monoculture systems, at the next plots. The forest species utilized were guayacán blanco (Cybistax donnell-smithii Rose), laurel prieto (Cordia megalantha Chadat), fernansánchez (Triplaris cumingiana Wedd) and teca (Tectona grandis L. F.). The intention was to compare the agroforestry systems and the monoculture system in both components trees and coffee. The production by hectare of coffee was major in monoculture system. The incidence (\%) of mining of the coffee (Perileucoptera coffeella Green) was low in the two systems; also, there were no significant differences among both coffee plantation systems. The volume of the forest species was major in the agroforestry systems. According to the evaluation of the land efficiency usage, anyone of the agroforestry systems evaluated is better than the pure cultivations of these species.
\end{abstract}

Key words: Association, monoculture system, forest species, coffee.

\section{INTRODUCCIÓN}

$\mathrm{L}$ os sistemas agroforestales (SAF) son modelos de

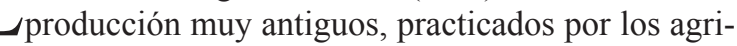
cultores de todo el mundo y que, talvez, coinciden con los orígenes de la agricultura. El primer esfuerzo por racionalizar el sistema fue realizado por los ingleses, mediante la introducción del sistema Taungya, como un método de cultivar la teca (King, 1987). La asociación de árboles con los cultivos ha sido sugerida como una forma de mejorar el sistema tradicional de corta y quema en el trópico húmedo (Jurion y Henry, 1969; Oladokun, 1990). Los sistemas agroforestales bien manejados pueden ayudar a solucionar los problemas de

Recibido: Junio, 2009. Aceptado: Octubre, 2009.

Publicado como ARTÍCULO en Ciencia y Tecnología 2(2): 29-34. 2009 rentabilidad del café ( $C$. arabica L.), ya que además de modificar positivamente el microambiente del cultivo y mejorar el suelo incrementando la materia orgánica y los nutrimentos (Beer et al., 1998), pueden reducir los costos de producción y el uso de insumos químicos, $\mathrm{y}$ proveer ingresos adicionales al productor (Somarriba, 1999).

El café ha sido tradicionalmente cultivado bajo un dosel de sombra de árboles (Perfecto et al., 1996). Estos sistemas se han reportado como una buena alternativa cuando el uso de monocultivos no es económicamente factible debido a la alta cantidad de insumos externos que demandan (Montagnini, 1992). Con la introducción de árboles maderables o frutales se regula las condiciones de luz, se puede suplir parte de los 
requerimientos de nutrientes del cultivo, diversificar la producción, y proveer un seguro contra fluctuaciones de los precios en el mercado (Montagnini, 1992; Fassbender, 1993). La sombra bien regulada puede reducir la incidencia de ciertas plagas y contribuir a una larga vida útil de la plantación de café (Staver et al., 2001; Vaast et al., 2002). Además, las especies forestales utilizadas como sombra tienen gran importancia comercial en el Litoral ecuatoriano, pues son muy codiciadas como madera de buena calidad, utilizadas en ebanisterías y construcciones especialmente.

El cultivo del café en el Ecuador se ha practicado desde hace algunos años, tanto en la región del Litoral como en la Amazonía. Por lo general, el café se ha cultivado en asociación con otras plantas que le proveen de sombra. A pesar de los beneficios que ofrecen estos sistemas, muchos agricultores están cambiando el sistema tradicional del cultivo de café por plantaciones industriales con muy poca o ninguna sombra, debido a la sugerencia de algunas agencias de servicio de extensión agrícola, que recomiendan este tipo de plantaciones industriales con el fin de obtener los más altos rendimientos (Perfecto et al., 1996). Ante esta situación la Unidad de Investigación Científica y Tecnológica (UICYT) de la UTEQ, pretende ofrecer información técnica que le permita al agricultor optimizar la producción agroforestal. En esta investigación se planteó como objetivos: determinar el desarrollo de las especies forestales y la producción del café en asociación y en monocultivo, realizar una evaluación fitosanitaria del cultivo de café, y evaluar el uso eficiente de la tierra en términos de producción e ingresos.

\section{Materiales y MÉTOdos}

$\mathrm{E}$ 1 sistema agroforestal con café fue establecido en la finca experimental "La Represa" de la Universidad Técnica Estatal de Quevedo, ubicada en el cantón Quevedo, provincia de Los Ríos. Esta área se encuentra entre las coordenadas geográficas: $01^{\circ} 03^{\prime} 24^{\prime \prime}$ de latitud sur y $79^{\circ} 24^{\prime} 55^{\prime \prime}$ de longitud oeste. Corresponde a la zona ecológica bosque húmedo tropical (bh-T) (Holdrige, 1978). Presenta una altitud media de $90 \mathrm{msnm}$, precipitación anual de $2178 \mathrm{~mm}$, temperatura media de $24.5^{\circ} \mathrm{C}$, heliofanía de $7.52 \mathrm{~h} \mathrm{~d}^{-1}$. La topografía es relativamente plana, suelo franco - arcilloso con $\mathrm{pH}$ de 6.5 -7.0 y humedad relativa de $84 \%$.

El experimento se estableció en el año 1997 y consistió en nueve tratamientos, cuatro en sistema agroforestal (especies forestales + café) y cinco en monocultivo (4 especies forestales y café solo); en el sistema agroforestal se incluyó el plátano como sombra temporal. Las parcelas fueron instaladas bajo un diseño de bloques al azar con tres repeticiones. Para las compara- ciones entre medias de tratamientos, se utilizó la prueba de Tukey a un nivel de significancia de 0.05 (Padrón, 1996). Las especies forestales utilizadas en esta investigación fueron: Guayacán blanco (T. donnell- smithii Rose), Laurel prieto (C. megalantha S.F. Blake), Teca (T. grandis L. f.) y Fernansánchez (T. cumingiana Fisch. $\&$ C.A. Mey. ex C.A. Mey). Las especies agrícolas fueron café (C. arabica L.) y plátano (Musa $s p$ ). En las especies forestales se evaluaron las variables: diámetro y altura; en el café la producción e incidencia de plagas, y en el plátano se registró la producción. Las cuatro especies forestales y el café fueron propagadas en el vivero de la UICYT. Para determinar si los Sistemas Agroforestales son más eficientes que los monocultivos se calculó la RET (Razón equivalente de la tierra) y la REI (Razón Equivalente de los Ingresos) (Guamán, 1981).

A todos los componentes del sistema se aplicó, en el fondo del hoyo al momento de la siembra, una combinación de nitrógeno, fósforo y potasio (10-30-10) en dosis de $30 \mathrm{~g}$ por planta; posteriormente, en el primer año, se aplicó una fertilización con urea, en dosis de 30 g por planta (en media luna). Para controlar el ataque de esqueletizadores de las hojas y las arrieras (Atta $s p$ ), en fernansánchez y café, se utilizó Nuvacrón 40EC, en dosis de $1.5 \mathrm{~cm}^{3}$ por 1 litro de agua. Para el control de nemátodos y picudo (Coleoptera: Curculionidae) en el plátano se aplicó Furadan $10 \mathrm{G}$ en dosis de 5 g por planta. Además, en el café se aplicó abono foliar (Nitrofosca) en dosis de $300 \mathrm{~g}$ por $20 \mathrm{~L}$ de agua en los meses de julio y septiembre del primer año.

\section{RESUltados Y DISCUSIÓN}

los cinco años de establecido el ensayo las espe$A_{\text {cies forestales presentaron diferencias estadísticas }}$ $(\mathrm{P} \leq 0.05)$ en las variables evaluadas. Los árboles presentaron mayor crecimiento en diámetro en el sistema agroforestal. El mayor incremento en diámetro, altura y volumen se registró en teca, tanto en asociación con café como en monocultivo (Cuadro 1). El laurel prieto presentó el menor incremento en diámetro, altura $\mathrm{y}$ volumen, tanto en asociación como en monocultivo (Cuadro 1), coincidiendo con Hernández et al. (1997), quienes señalan que en Costa Rica, el laurel obtuvo el menor crecimiento dentro de un sistema agroforestal con café; mientras que Montenegro et al. (1997) reporta mayor crecimiento en diámetro de C. alliodora (Laurel blanco), Cedrela odorata L. (Cedro) y Eucaliptus deglupta Blume (Eucalipto) en relación a otras especies asociadas con café. 
Cuadro 1. Diámetro, altura, volumen y el incremento medio anual (IMA) en cuatro especies forestales, a los cinco años de edad, asociadas con café y en monocultivo en el Cantón Quevedo.

\begin{tabular}{llllllllll}
\hline \multicolumn{1}{c}{ Tratamientos } & \multicolumn{1}{c}{ Diámetro (cm) } & IMA $^{\mathbf{1}}(\mathbf{c m})$ & Altura $(\mathbf{c m})$ & IMA $(\mathbf{c m})$ & Volumen $\left(\mathbf{m}^{\mathbf{3}}\right)$ & IMA $(\mathbf{c m})$ \\
\hline Asociación & & & & & & & & & \\
\hline Guayacán blanco + café & 21.80 & $\mathrm{ab}$ & 4.21 & 14.89 & $\mathrm{ab}$ & 2.85 & 0.39 & $\mathrm{~b}$ & 0.08 \\
Laurel prieto + café & 16.20 & $\mathrm{~d}$ & 3.11 & 10.87 & $\mathrm{c}$ & 2.11 & 0.16 & $\mathrm{c}$ & 0.03 \\
Teca + café & 26.50 & $\mathrm{a}$ & 5.12 & 16.23 & $\mathrm{a}$ & 3.17 & 0.64 & $\mathrm{a}$ & 0.13 \\
Fernansánchez + café & 21.00 & $\mathrm{bc}$ & 4.02 & 15.72 & $\mathrm{a}$ & 3.03 & 0.38 & $\mathrm{~b}$ & 0.08 \\
\hline Monocultivo & & & & & & & & \\
\hline Guayacán blanco & 12.20 & $\mathrm{de}$ & 2.27 & 13.00 & $\mathrm{bc}$ & 2.50 & 0.11 & $\mathrm{c}$ & 0.02 \\
Laurel prieto & 11.10 & $\mathrm{e}$ & 2.07 & 10.90 & $\mathrm{c}$ & 2.12 & 0.07 & $\mathrm{c}$ & 0.02 \\
Teca & 16.90 & $\mathrm{~cd}$ & 3.24 & 17.00 & $\mathrm{a}$ & 3.33 & 0.27 & $\mathrm{bc}$ & 0.05 \\
Fernansánchez & 13.30 & $\mathrm{de}$ & 2.46 & 15.24 & $\mathrm{ab}$ & 2.93 & 0.15 & $\mathrm{c}$ & 0.03 \\
\hline
\end{tabular}

${ }^{1} \mathrm{IMA}=$ Incremento Medio Anual

Se registraron bajos porcentajes de incidencia del minador ( $P$. coffeella Green) en todos los tratamientos; la menor incidencia se encontró en la asociación "laurel prieto + café". No hubo diferencia estadística entre el café asociado y en monocultivo (Figura 1), confirmando lo reportado por Monterrey et al. (2001), quienes obtuvieron un resultado similar en Nicaragua al evaluar el comportamiento de este insecto en sistemas agroforestales con café. El ataque de la broca del café (Hypothemus hampei Ferr.) fue bajo y no presentó diferencias $(\mathrm{P} \leq 0.05)$ entre los dos sistemas. Al contrario Félix et al. (2004) señala que obtuvo menor infestación de broca en plantaciones de café a pleno sol, al compa- rarlos con sistemas agroforestales de Eugenia jambos L. y Gliricidia sepium (Jacq.) Kunth ex. Griseb $+C$. arabica L.; este resultado diferente se puede atribuir a los árboles utilizados como sombra.

En esta investigación se registró una mínima incidencia de la roya (Hemileia vastatrix B.), tanto en asociación como en monocultivo, coincidiendo con Samayoa y Sánchez (2000), quienes no encontraron diferencias en la incidencia y severidad de la roya al evaluar cafetales bajo sombra y en monocultivo, en Costa Rica; por su parte, la menor incidencia de otras enfermedades las relaciona con un menor porcentaje de sombra.

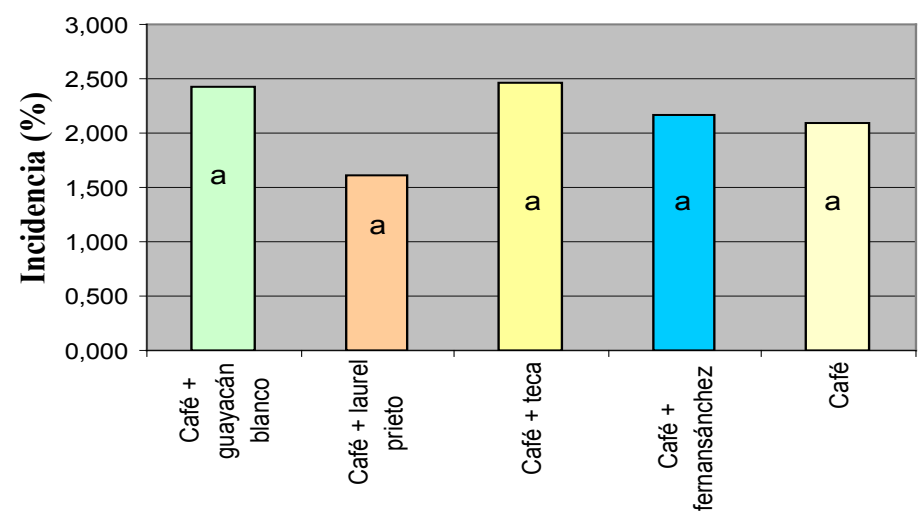

Figura 1. Incidencia (\%) del minador en la hoja del café asociado con cuatro especies forestales y en monocultivo en el Cantón Quevedo. 
La producción del café presentó diferencias estadísticas $(\mathrm{P} \leq 0.05)$. En el sistema asociado los promedios más altos se registraron en los tratamientos "café + laurel prieto" con $2,113.82 \mathrm{~kg} \mathrm{ha}^{-1} \mathrm{a}^{-1} \mathrm{y}$ "café + teca" con $2,093.53 \mathrm{~kg} \mathrm{ha}^{-1} \mathrm{a}^{-1}$; el tratamiento con menor producción fue café + guayacán blanco con $908.49 \mathrm{~kg} \mathrm{ha}^{-1} \mathrm{a}^{-1}$. La producción del café fue mayor en el sistema de monocultivo con $3,977.20 \mathrm{~kg} \mathrm{ha}^{-1} \mathrm{a}^{-1}$ frente a los valores indicados para el sistema agroforestal. Esto coincide con Félix et al. (2004) y Hernández et al. (1997), quienes también encontraron, en Nicaragua y Costa Rica, respectivamente; una mayor producción de granos de café en plantaciones a plena luz y manifiestan que se debe a la mayor densidad del café en esas condiciones.

La producción media anual de plátano no presentó diferencias estadísticas $(\mathrm{P} \leq 0.05)$. La producción media anual de madera varió entre especies y entre sistemas, con valores entre 19.68 a 78.23 en los SAF y 20.72 a $75.32 \mathrm{~m}^{3} \mathrm{ha}^{-1}$ en los monocultivos (Cuadro 2). La teca obtuvo la mayor producción de madera con $78.23 \mathrm{~m}^{3} \mathrm{ha}^{-1}$ en asociación, de igual manera en monocultivo con $75.32 \mathrm{~m}^{3} \mathrm{ha}^{-1}$; mientras el laurel obtuvo la menor producción de madera, tanto en asociación como en monocultivo (Cuadro 2).

Cuadro 2. Producción media anual del café, plátano y madera en el sistema agroforestal asociación de cuatro especies forestales con café en el Cantón Quevedo.

\begin{tabular}{|c|c|c|c|}
\hline Tratamientos & $\begin{array}{c}\text { Café } \\
\left(\mathrm{Kg}^{\prime} / \mathbf{h a}^{-1}\right)\end{array}$ & $\begin{array}{c}\text { Plátano } \\
\left(\text { Racimos ha }^{-1} \text { año-1 }^{-1}\right)\end{array}$ & $\begin{array}{r}\text { Madera } \\
\left(\mathbf{m}^{3} \mathbf{h a}^{-1}\right)\end{array}$ \\
\hline \multicolumn{4}{|l|}{ Asociación } \\
\hline Guayacán blanco + café & 908.49 a & $60.00 \mathrm{a}$ & 48.46 \\
\hline Laurel prieto + café & $2,113.82 \quad b$ & $65.70 \quad \mathrm{a}$ & 19.68 \\
\hline Teca + café & $2,093.53 \quad b$ & 63.30 & 78.23 \\
\hline Fernansánchez + café & $1,734.12 \mathrm{c}$ & $60.00 \mathrm{a}$ & 46.99 a \\
\hline \multicolumn{4}{|l|}{ Monocultivo } \\
\hline Café solo & $3,977.20 \quad \mathrm{~d}$ & & \\
\hline Guayacán blanco & & & 30.24 \\
\hline Laurel prieto & & & 20.72 \\
\hline Teca & & & 75.32 \\
\hline Fernansánchez & & & $42.00 \mathrm{a}$ \\
\hline
\end{tabular}

El beneficio neto de todo el sistema, fue mayor para el tratamiento "café + teca" $\left(3,033.81\right.$ USD ha $\left.^{-1}\right)$ frente al "café solo" (1,070.55 USD ha-1). Comparando los sistemas estudiados, la Relación Beneficio - Costo es mejor en los monocultivos (Cuadro 3), lo que coin- cide con Hernández et al. (1997), quienes reportan un mayor margen de utilidad en plantaciones puras de café, al compararlo en asociación con $C$. alliodora (Laurel blanco).

Cuadro 3. Ingresos, egresos y beneficio neto (\$) del café, plátano y madera en el sistema agroforestal asociación de cuatro especies forestales con café y monocultivos en el Cantón Quevedo.

\begin{tabular}{lcccc}
\hline \multicolumn{1}{c}{ Tratamientos } & $\begin{array}{c}\text { Egresos } \\
\text { (USD) }\end{array}$ & $\begin{array}{c}\text { Ingresos } \\
\text { (USD) }\end{array}$ & $\begin{array}{c}\text { Beneficio Neto } \\
\text { (USD) }\end{array}$ & $\begin{array}{c}\text { Relación } \\
\text { Beneficio-Costo }\end{array}$ \\
\hline Asociación & & & & \\
\hline Guayacán blanco + café & $1,146.42$ & $2,578.93$ & $1,432.51$ & 1.25 \\
Laurel prieto + café & $1,146.42$ & $2,040.97$ & 894.56 & 0.78 \\
Teca + café & $1,146.42$ & $4,180.23$ & $3,033.81$ & 2.65 \\
Fernansánchez + café & $1,146.42$ & $2,277.36$ & $1,130.94$ & 0.99 \\
\hline Café & 743.45 & $1,814.00$ & $1,070.55$ & 1.44 \\
\hline Guayacán blanco & 332.32 & $3,268.73$ & $2,936.42$ & 8.84 \\
Laurel prieto & 332.32 & $1,432.12$ & $1,099.80$ & 3.31 \\
Teca & 332.32 & $9,682.01$ & $9,349.69$ & 28.13 \\
Fernansánchez & 332.32 & $2,665.65$ & $2,333.33$ & 7.02 \\
\hline
\end{tabular}


De acuerdo a la RET, cualquiera de las asociaciones es más eficiente que los monocultivos bajo estudio, en términos de producción. Sin embargo la mejor RET obtenida fue con "Guayacán + café" comparada al resto de asociaciones (Cuadro 4). Somarriba (1994) coincide con que los SAF son una forma eficiente de uso de la tierra, habiendo obtenido valores RET de 1,103.00 en sistemas agroforestales con cacao- plátano -laurel. En términos de ingresos (REI) la asociación Guaya- cán + café, así mismo fue la mejor opción, mientras la asociación de laurel prieto + café no es recomendable; siendo mejor en este caso, su cultivo en plantaciones puras. Cabe indicar, que estos resultados se deben a que la producción de las otras especies fue similar tanto en asociación como en monocultivo; mientras que la producción del guayacán fue más del doble en asociación $\left(48.46 \mathrm{~m}^{3}\right)$ en relación a su cultivo puro $\left(20.72 \mathrm{~m}^{3}\right)$.

\begin{tabular}{|c|c|c|}
\hline Asociación & RET & REI \\
\hline Guayacán blanco + café & 1.83 & 2.18 \\
\hline Laurel prieto + café & 1.48 & 0.95 \\
\hline Teca + café & 1.56 & 1.44 \\
\hline Fernansánchez + café & 1.55 & 1.68 \\
\hline
\end{tabular}

\section{Conclusiones}

$\mathrm{L}$ a producción de café por hectárea fue mayor bajo el sistema en monocultivo, frente al sistema agroforestal, debido a la mayor densidad del café en plantación pura.

La incidencia del minador del café no fue significativa y los daños reportados fueron de poca importancia. Así mismo, la incidencia de la broca y de la roya fueron muy bajas.

El volumen de madera varió entre especies y entre sistemas de producción, los valores más altos en diámetro y volumen se registraron en las asociaciones, a pesar de esto no se presentaron diferencias significativas en la producción por unidad de superficie debido a la mayor densidad de las especies forestales en monocultivo.

La equivalencia de la tierra indica que las asociaciones de especies forestales con café, son una mejor alternativa, en términos de producción. En términos de ingresos la asociación de laurel prieto + café no es recomendable, siendo más indicado su cultivo en plantaciones puras.

\section{Literatura CiTADA}

Beer, J., R. Muschler, D. Kass y E. Somarriba. 1998. Shade management in coffee and cacao plantation. Agroforestry systems 38: 139-164.

Fassbender, H.W. 1993. Modelos edafológicos de Sistemas Agroforestales. Serie de materiales de enseñanza No. 29. 2da. Edición. CATIE-GTZ, Turrialba, Costa Rica, 491p.
Félix, D., F. Guruhay y J. Beer. 2004. Incidencia de la broca (Hypothenemus hampei) en plantas de café a pleno sol y bajo sombra de Eugenia jambos y Gliricidia sepium en San Marcos, Nicaragua.

Guamán, R., 1981. Rendimiento físico y económico del frijol (Phaseolus vulgaris L.) y maíz (Zea mays L.) sembrados solos y en asociación. Tesis M.C. Chapingo, MX, CP. $122 \mathrm{p}$.

Hernández, O., J. Beer y H. Platen. 1997. Rendimiento de café (Coffea arabica cv Caturra), producción de madera (Cordia alliodora) y análisis financiero de plantaciones con diferentes densidades de sombra en Costa Rica. Agroforestería de las Américas 4 (12): 8- 13

Holgrige, L., 1987. Ecología basada en las zonas de vida. San José, Costa Rica, IICA. 216 p.

Jurion, F. y J. Henry. 1969. Can primitive farmin be modernized?. Brussels, Institute National pour l'Etude Agronomique du Congo. 445 p.

King, KFS. 1987. The history of agroforestry. In Steppler, HA; Nair, PKR. (eds.). Agroforestry; a decade of development. Nairobi, Kenia, ICRAF. p. 3-11.

Montagnini, F. 1992. Sistemas Agroforestales. Principios y aplicaciones en los trópicos. 2da. edición. Costa Rica, Organización para Estudios Tropicales. $622 \mathrm{p}$.

Montenegro, J., G. Ramírez y MH. Blanco. 1997. Evaluación del establecimiento y crecimiento inicial de seis especies maderables asociadas con café. Agroforestería en las Américas 4 (13): 14-20.

Monterrey, J., D. Suárez y M. González. 2001. Comportamiento de insectos en sistemas agroforestales 
con café en el Pacífico Sur de Nicaragua. Agroforestería de las Américas. 8 (12): 15-21 p.

Oladokun, M. 1990. Tree crop based agroforestry in Nigeria: a checklist of crops intercropped with cocoa. Agroforestry systems 11: 227-241.

Padrón, E. 1996. Diseños Experimentales con aplicación a la agricultura y la ganadería. México. Trillas. $215 \mathrm{p}$.

Perfecto, I., RA. Rice, R. Greenberg y M Van der Voort. 1996. Shade coffee: a disappearing refuge for biodiversity. Bioscience 46 (8): 598- 608.

Somarriba, E. 1994. Sistema agroforestal con CacaoPlátano- Laurel. Agroforestería de las Américas 1 (4): 22-24.

Somarriba, E. 1999. Sustainable timber production from uneven-aged shade stands of Cordia alliodora in small coffee faros. Agroforestry systems 10: 253- 263.
Staver, C., F. Guharay, D. Monterosso y R. Muschler. 2001. Designing pest- suppressive multistrata perennial crop system: shade- grown coffee in Central America. Agroforestry systems 53: 151-170.

Somayoa, J. y V. Sánchez. 2000. Importancia de la sombra en la incidencia de enfermedades en café orgánico y convencional en Paraíso, Costa Rica 7 (26): 34- 36.

Vaast, P., M. Génard y J. Dauzat. 2002. Modeling the effects of fruit load, shade and plant water status on coffee berry growth and carbon partitioning at the branch level. In Dejong, TM. Ed. Proceedings of the Sixth International Symposium in Fruit Research and Orchard Management. Acta Horticulturae 584: 57-62. 